\title{
The effects of preoperative imaging stability studies on intraoperative correction, postoperative observation, and clinical efficacy of adult lumbar spondylolisthesis treatment
}

Jie Jiang

Guangxi Medical University

Chong Liu

Guangxi Medical University First Affiliated Hospital

Guangping Zhang

Yulin City First Hospital Yulin Branch

Guoyong Xu

Guangxi Medical University

Tuo Liang

Guangxi Medical University

Chaojie Yu

Guangxi Medical University

SHian Liao

Guangxi Medical University

Zide Zhang

Guangxi Medical University

Zhaojun Lu

Guangxi Medical University

\section{Zequn Wang}

Guangxi Medical University

Jiarui Chen

Guangxi Medical University

Tianyou Chen

Guangxi Medical University

Hao Li

Guangxi Medical University

Xinli Zhan ( $\sim$ zhanxinli_215@163.com )

Guangxi Medical University https://orcid.org/0000-0002-4599-1544 
Research article

Keywords: Spondylolisthesis, stability, preoperative imaging, pedicle screws, complication

Posted Date: August 26th, 2020

DOI: https://doi.org/10.21203/rs.3.rs-58700/v1

License: (c) (i) This work is licensed under a Creative Commons Attribution 4.0 International License. Read Full License 


\section{Abstract \\ Background}

The aim of this study is to explore the use of preoperative imaging stability studies of adult lumbar spondylolisthesis and it's effects on intraoperative correction, postoperative observation, and clinical efficacy.

\section{Methods}

We retrospectively analyzed a total of 104 patients diagnosed with lumbar spondylolisthesis who underwent Posterior lumbar interbody fusion surgery between 2011 and 2014. The qualified patients were divided into two groups; group A (study/unstable group: 52 cases) and group B (control/stable group: 52 cases). Group A was further divided into group A1 (slip instability: 27 cases) and group A2 (angular instability: 25 cases). The preoperative lumbar sagittal plane translation distances and segmental angle of the preoperative lateral X-rays, hyperflexion X-rays, and hyperextension X-rays were measured and compared with their third-day postoperative lateral X-ray, and slip rate. The occurrences of intraoperative or postoperative complications, one-month postoperative follow-up data, including JOA, and VAS scores were access to evaluate the clinical efficacy of the treatment.

\section{Results}

Satisfactory postoperative radiographic correction was recorded in all groups. Better correction and correction was observed in group $\mathrm{A} 1$ patients and group $\mathrm{B}$ patients who used puller screws compared to those who used normal screws $(P<0.05)$. However, patients in group $A 2$ who used normal pedicle screws had similar correction as those who used puller screws. The preoperative VAS scores showed that low back and lower extremity pain were higher in A1 and A2 groups compared to group B. However, they were lower one month postoperative. The preoperative JOA scores for the unstable groups (group A1 and A2) were lower than their stable counterpart but higher during the first month postoperative. Our analysis of postoperative complications also revealed no statistically significant difference between groups A1 and $B$, and groups $A 2$, and $B$.

\section{Conclusions}

Preoperative imaging stability for adult lumbar spondylolisthesis does not affect intraoperative correction. The use of puller pedicle screws in patients with unstable or stable segmental slippage could achieve better correction and correction than with normal pedicle screws. The preoperative symptoms of patients with unstable segments were worse than their stable counterparts; however, their postoperative recovery was quicker in the first month postoperative. 


\section{Background}

Lumbar spondylolisthesis, defined as a series of clinical syndromes caused by the displacement of an upper vertebral body relative to a lower vertebral body. Common symptoms include lower back pain, worsened by prolonged standing or sitting, but relieved by bed rest in early stages. As the disease progresses, other symptoms such as lower back pain with intermittent claudication, sciatica, and lower limbs radicular pain and numbness ${ }^{[1],[2]}$ may develop. Patients with lumbar spondylolisthesis of lower lumbar spine make up the majority of spondylolisthesis cases, and most cases predominantly involve females than males. According to Ravindra VM et al., there are an estimated 39 million cases of lumbar spondylolisthesis annually ${ }^{[3]}$. This high number of annual cases necessitate better diagnostic efficacy and specificity as well as better treatment methods.

Khor S et al. Reported that surgical fusion of an unstable lumbar spine can successfully correct lumbar spine stability ${ }^{[4]}$. Traditionally, conservative treatment for lumbar spondylolisthesis is considered for patients with mild symptoms, and surgical treatment recommended for patients with severe symptoms that affect their daily life, and those that failed conservative treatment ${ }^{[5]}$. Improvements in imaging technologies in combination with other medical diagnostic methods have led to improved preoperative diagnostic efficacy and specificity, enabling patient-specific treatment plans. There are currently few reports on the preoperative imaging evaluation of the stability of a diseased lumbar segment and its effect on intraoperative and postoperative outcomes. The aim of this study is to retrospectively analyze available clinical data in order to investigate whether such stability of a diseased lumbar segment could affect intraoperative correction, postoperative complications, and overall clinical outcomes in adult lumbar spondylolisthesis patients.

\section{Methods}

\section{Research Object}

We retrospectively analyzed the data of patients diagnosed with lumbar spondylolisthesis who underwent Posterior lumbar interbody fusion(PLIF) surgery in our hospital between November 2011 and June 2014. The inclusion criteria were: 1 . Patients $\geq 18$ years with the confirmed diagnosis of lumbar spondylolisthesis $₫ 2$. Patients with complete preoperative radiographic lateral lumbar X-ray, as well as hyperextension and hyperflexion X-ray; 3 . Patients with the unstable lumbar spine on lateral X-ray film with a measured segmental sliding/dislocation of $>3 \mathrm{~mm}$; 4 . Patients who underwent PLIF surgery; 5 . Patients with complete follow-up data for $>18$ months, with complete follow-up radiographic data. This study exclusion criteria were: 1 . Patients who underwent multiple segment fusion $(n>2) ; 2$. Patients with severe osteoporosis and vertebral compression fractures; 3 . Patients with the previous history of lumbar spine surgery; 4. Patients who refused surgery or transferred to other departments for treatment; 5. Patients with the fusion of preoperative lesions. 
This study was approved by the Ethical and review committee of the First affiliated hospital of Guangxi Medical University. The study conforms to the Helsinki declaration; however, since patient data were anonymized, informed consent was unnecessary.

\section{Collection of cases data}

The clinical data of all patients who passed the inclusion criteria including baseline clinical data, preoperative and postoperative imaging indicators, intraoperative fixation and correction data including type of pedicle screws used, pre- and postoperative JOA and VAS scores, and postoperative complications status were collected for statistical analysis.

\section{Grouping methods and Imaging analysis indexes.}

The qualified patients were divided into two groups; group A (study/unstable group: 52 cases) and group $B$ (control/stable group: 52 cases). According to the sagittal plane sliding range of lumbar hyperflexion and hyperextension X-ray film patients with $>3 \mathrm{~mm}$ of sagittal sliding were classified as group A1 (slip instability: 27 cases); those with the angle of rotation range $>10^{\circ}$ were classified as unstable angle group A2 (angular instability: 25 cases) ${ }^{[6]}$; whiles patients with normal sagittal sliding and normal angle of rotation were classified as the control group or stable group (group B).

Preoperative lateral lumbar, lumbar hyperflexion and hyperextension radiographs, and third day postoperative lateral lumbar radiographs were imported into the Surgimap (Version: 2.2.9.8) software for

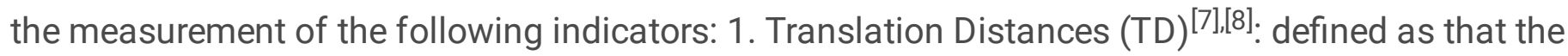
distance between Intersection of a vertical line drawn from the inferior posterior angle of a slipped vertebral body segment relative to the endplate of the inferior vertebral body segment and the superior posterior angle of the inferior vertebral body; 2 . Segment angle(SA): defined as the angle formed by the Lower endplate line of the vertebral body of a spondylolisthetic segment and the upper endplate line of the lower vertebral body of the same segment; 3. Slip rate ${ }^{[9],[10]}(\mathrm{SR})$ : defined as the percentage of slip distance to the width of the sagittal plane of the inferior vertebral body; 4 . Slip distance correction = preoperative slip distance-postoperative slip distance; 5 . Segment angle correction = preoperative segment angle- postoperative segment angle; 6 . Correction of slippage rate = preoperative slippage ratepostoperative slippage rate. The schematic diagram is shown in Figure 1.

\section{Surgical technique}

All of the patients enrolled in this study underwent PLIF surgery ${ }^{[11]}$. This operation involves extensive decompression to relieve compression on the dural sac and nerve root, and loosen the adhesion tissue around the nerve root; the resection of nucleus pulposus of the intervertebral disc, and the distractor used in open correction or a combination of distraction and pedicle screws used for correction, However, the aim is not to achieve complete anatomical correction. Patients on a postoperative day (POD) 2-4 undergo orthotopic and lateral X-ray, and after no abnormalities were found, instructed to wears lumbar braces 
during ambulation for a period of at least three months whenever they get out of bed. Patients were advised to return for follow-up on $1^{\text {st }}, 3^{\text {rd }}, 6^{\text {th }}$ month, and $1^{\text {st }}$ year after discharge.

\section{Statistical software and methods}

$\chi^{2}$ or Fisher exact tests were used to test the baseline information of each group. Independent sample rank-sum tests were used to test the preoperative and postoperative imaging indexes, while an independent sample t-test was used to analyze the preoperative, first month postoperative, and last follow-up JOA and VAS scores. All statistical analyses were performed using SPSS 25.0 software (IBM, USA), with a $P<0.05$ considered statistically significant.

\section{Results}

\section{Baseline information.}

The average follow-up period of the 104 patients included in this study was 32 months. According to the grouping method, eligible patients were divided into study and control groups: group A (study/unstable group: 52 cases), and group B (control/stable group: 52 cases). Group A was further divided into group A1 (slip instability: 27 cases) and A2 (angular instability: 25 cases). The clinical characteristics of groups A1 and A2 were compared with group B, respectively, and their baseline data tested using the $\chi^{2}$ test or Fisher exact probability method. Tested data included screw type, number of fusion segments, operation time, follow-up duration, among others. Results were considered not to be statistically different when $\mathrm{P}>$ 0.05. See Table 1-1 and Table 1-2 for details.

\section{Preoperative imaging parameters.}

Among the cases reviewed, the preoperative slip distance and slip rate were found to be higher in patient in group $A 1$ in comparison to group $B$; however, the difference was not statistically significant $(P>0.05)$. Also, the preoperative segment angle difference among patients in group A2 were higher compared to those in group $B$, the difference however, was not statistically significant $(P>0.05)$. The detailed results are shown in Figure 2.

\section{Correction of slippage reset.}

The comparison of the preoperative and POD 3 lumbar lateral X-rays revealed no statistically significant difference in the slippage correction rate, segment angle correction, and slip distance between patients in group $A 1$ and those in group $B(P>0.05)$. The comparison of the average values of the two indexes (slippage rate correction and slip distance correction) between groups $\mathrm{A} 2$ and $\mathrm{B}$ revealed that the indexes in group $A 2$ were higher than those in group $B$. On the contrary, the average segmental angle of patients in group $B$ were higher than those in group $A 2$. The differences among the three indicators between the two groups, however, were not statistically significant $(P>0.05)$. See Figure 3 for details.

\section{Comparison of the pedicle screw typesused for reductionand correction.}


Data of patients in the three groups (A1, A2, and B) were analyzed in accordance with the type of pedicle screw used (normal screws or puller screws) for the correction of spondylolisthesis. Patients in groups A1 and $\mathrm{B}$, who used puller pedicle screws, achieved better correction and correction than patients who used normal pedicle screws. There was a statistically significant difference between the slippage correction and slip distance correction rates. However, there was no statistically significant difference in the segmental angular correction rate. There was no statistically significant difference in the correction rates of spondylolisthesis, segmental angle correction, and sliding distance correction in group A2 patients who used normal pedicle screws or puller screws. See Figure 4 for details.

\section{JOA scores analysis}

The total JOA score for the study population was 29 points, with the lowest score signifying severe dysfunction ${ }^{[12]}$. The postoperative improvement rate was calculated by subtracting the pretreatment score from the posttreatment score, then dividing the outcome with the outcome of the 29 minus posttreatment score. We observed that the preoperative score for groups $A 1$ and $A 2$ were lower than that of group $B$, and the difference was statistically significant $(P<0.05)$; however, this outcome was reversed at one month postoperative in both groups ( $A 1$ and $A 2)$. The analysis of the last follow-up data revealed no statistically significant difference in the JOA scores between groups $A 1$ and $B$, as well as $A 2$ and $B$. The one month postoperative and last follow-up improvement rate for patients in group $A 1$ were higher than those in the $B$ group, and the difference was statistically significant $(P<0.05)$. There was a statistically significant difference between the improvement rate in group A2 when compared to group $B$ $(P<0.05)$, but there was no statistically significant difference in the improvement rate at the last follow-up $(P>0.05)$. See Figure 5 for details.

\section{VAS scores for lower back and limb pain}

The VAS scoring, which is used to evaluate the patient's pain severity subjectively, has a scoring system with 0 signifying no pain, and 10 for unbearable/severe pain. The average preoperative VAS scores for groups $A 1$ and $A 2$ were higher than that of group B. On the contrary, the average at one month postoperative was lower than group B. At the last follow-up, the average scores for patients in group $A 1$ were lower than those in group $B$, while that of group $A 2$ were higher than those in group $B$. At the last follow-up, the average scores for groups A1 and A2 were lower than those of group B. See Figure 6 for details.

\section{The occurrence of complications.}

Among the 104 cases reviewed, 58 patients developed temporary intestinal paralysis, and eight patients developed wound infection, seven patients developed CSF leakage, five patients developed nervous system-related injuries, while two patients developed urinary system-related complications. There were no statistically significant differences in the incidence of complications among the five main postoperative complications between groups $A 1$ and $B$, and groups $A 2$ and $B(p>0.05)$. See Figure 7 for details. 


\section{Discussion}

\section{Evaluation of the stability of lumbar spondylolisthesis}

The anatomy of the spinal segments of a healthy person allows for a limited physiological range of motion, including forwarding and backward bending, as well as lateral flexion and extension. This range of movements does not permit excessive movement and is dependent on the level of resistance from structures of spinal segments such as intervertebral discs, joints facets, muscles, and ligaments associated with the spine. At present, most researchers use the segment angle of a diseased segment and slip distance to evaluate postoperative stability of segmental spondylolisthesis, however, there is no general consensus on a fixed method. In this study, we used preoperative sagittal lumbar X-rays of spondylolisthesis patients; with hyperflexion and hyperextension $>3 \mathrm{~mm}$ or angular rotation $>10^{\circ}$ used as the criterion for assessing lumbar instability ${ }^{[13]}$. This criterion has been well accepted by other scholars $^{[14]}$. Okuda S. et al. Reported that deformation of facet joints may lead to accelerated degradation of the spinal stabilization, and to a certain extent, affect the progress of degenerative spondylolisthesis ${ }^{[15]}$. It has been reported that degenerative lumbar spondylolisthesis could naturally lead to spontaneous stabilization ${ }^{[16]}$. These above studies, therefore, demonstrate that although the methods of assessing the stability of lumbar spondylolisthesis are not clear, there could still be varying results as different patients produce different degrees of inherent instability. Segment instability is one of the critical indicators for operative lumbar spine fusion. In this study, all patients underwent posterior lumbar interbody fusion surgery (PLIF). We compare group $A 1$ with group $B$, and group $A 2$ with group $B$, however, there was no statistically significant difference in the baseline data between groups $A 1, A 2$, and $B$ groups. There was also no statistically significant difference between the preoperative imaging indicators. Our results also revealed that there was no statistically significant difference between preoperative and postoperative imaging indexes (segment angle, slippage correction rate, and slip distance correction rate) $(P>0.05)$. Therefore, we believe that preoperative stability observed in preoperative imaging of spinal lesions has no obvious effect on intraoperative correction of lumbar spondylolisthesis treatment. Our results, therefore, support previous studies that PLIF surgery can be successfully used to improve patients with lumbar spine instability.

\section{Correction of Lumbar spondylolisthesis}

In patients with low-grade spondyloarthritis presenting with radiation sickness and or pseudo claudication, a trial of conservative treatment may be considered; if conservative treatment does not resolve the problem, surgical options may include decompression alone or decompression and fusion ${ }^{[17]}$. However, further studies have demonstrated that decompression alone increased the risk of reoperation for lumbar spondylolisthesis ${ }^{[18]}$. However, there still exists controversies over whether slippage correction may be necessary for spondylolisthesis. Lian et al., reported that PLIF surgery and pedicle screw fixation successfully with or without correction resulted in the good postoperative outcome, however, deliberate correction with better imaging results did not necessarily correlate to better clinical outcomes ${ }^{[15]}$. Karsy M reported that postoperative pain relief and functional recovery were significantly better than those 
obtained via conservative treatment alone ${ }^{[19]}$. In summary, partial correction and internal fixation for slippage correction is more advantageous and could improve clinical efficacy, safety, and overall postoperative outcome. In this study, we observed that patients with unstable sliding segments and those with stable sliding segments obtained better correction and correction using puller pedicle screws than normal pedicle screws. However, patients with angular instabilities regardless of the use of the type of screws used resulted in similar correction and correction outcomes. Therefore, we believe that if conditions permit, puller pedicle screws should be the preferred choice, as it can improve the overall clinical efficiency. Also, extensive decompression of lateral recess, spinal canal, and nerve roots could help release pain and improve the overall postoperative functional outcome.

\section{Clinical efficacy}

In recent years, advancements in surgical treatment have resulted in increased efficiency of surgical treatment for adults' lumbar spondylolisthesis. Abdu WA reported in an 8-year follow-up study of surgical and non0surgical treatment for lumbar spondylolisthesis, surgically treated patients had better functional and pain improvements compared to non-surgically treated patients ${ }^{[20]}$. Methods commonly used in the clinical evaluation of postoperative efficacy include VAS score for pain (low back and leg pain), SF-36 scores, JOA scores, and ODI scores ${ }^{[12],[15,18],[21]}$. In this study, we used JOA scores and VAS scores for the assessment of postoperative pain. Our result demonstrates that patients in the unstable group had higher JOA scores for preoperative lower back pain than the stable group, on the contrary, they had VAS scores for lower back and leg pain. These results are similar to those reported in previous studies ${ }^{[22]}$. Our results also revealed that the first-month postoperative JOA scores for neurological function in the unstable group were higher than that of the stable group, while their VAS scores for lower back and leg pain during the same period were lower. These results show that neurological function and leg pain symptoms of patients in the unstable group improved faster than those in the stable group at one month postoperative. This may be due to the successful decompression and correction of patients in the unstable group, which may have resulted in improved nervous micro-environment, which necessitated early nerve root recovery and enhanced postoperative rehabilitation. On the contrary, the lack of decompression for patients in the stable group meant the nerve root compression still existed, hence delayed postoperative recovery. Therefore, PLIF surgery can effectively improve the short-term postoperative recovery of patients, and help relieve pain, neurological symptoms, and improve the overall clinical efficacy.

\section{The occurrence of complications}

In this study, there was no statistically significant difference in the incidence of postoperative complications between the stable and unstable groups. However, we observed that the incidence rate of incision infection was higher than previously reported ${ }^{[23]}$. Also, the incidence of postoperative reduced gastrointestinal motility was higher. When the lumbar spine was reviewed on the second to third days after surgery, a significant colon expansion was observed. These can be attributed to intraoperative pulling on nerve roots, however, it usually improves in most patients without the need for special 
treatment. The incidence of intraoperative neurological damage in this study was similar to previously reported studies ${ }^{[15]}$.

\section{Limitations of this study}

This study used a retrospective research methodology, and therefore there exists some level of recall bias. No group randomization was used. However, the same screening criteria and imaging measurement indicators were used. Also, the three groups' baseline data were similar, which helped reduce the level of bias. The study population was relatively small, as future multi-centered studies involving more cases may be needed for better clarification. Other factors that can influence intraoperative correction and postoperative recoveries, such as the degree of bone hyperplasia, joint facet anatomy, and intervertebral space characteristics, were not evaluated.

\section{Conclusion}

The preoperative segmental stability of a lesion segment does not affect the incidence of postoperative surgical complications. This is because, with sufficient intraoperative decompression, the preoperative stability of anatomical segments does not affect intraoperative correction. The use of puller pedicle screws produced better slippage correction and correction than normal pedicle screws. Patients with segmental instability are likely to report with symptoms such as lower back and leg pain and impaired neurological function than those with segmental stable, however, these symptoms rapidly improve one month postoperative, producing similar efficacy at the last follow-up those with stable segments.

\section{Abbreviations}

PLIF

Posterior lumbar interbody fusion

TD

Translation Distances

SA

Segment angle

$\mathrm{SR}$

Slip rate

POD

Patients on a postoperative day

\section{Declarations}

\section{Ethics approval and consent to participate}

This study was ethically reviewed by the First Clinical Affiliated Hospital of Guangxi Medical University. The approve number is 2020 (KY-E-054). 


\section{Consent for publication}

All co-authors were consulted and gave their consent for publication.

\section{Availability of data and materials}

Not applicable.

\section{Competing interests}

There are no conflicts of interest among any of the co-authors.

\section{Funding}

1.Youth Science Foundation of Guangxi Medical University, Grant/Award Numbers: GXMUYFY201712.

2.Guangxi Young and Middle aged Teacher's Basic Ability Promoting Project, Grant/Award Number: 2019 KY0119.

3.National Natural Science Foundation of China, Grant/Award Numbers: 81560359ه81860393.

\section{Author contributions}

Jie Jiang, Chong Liu, Guangping Zhang and Xinli Zhan designed the study. Chaojie Yu, Tuo Liang, Shian Liao, Guoyong Xu, Zide Zhang, Zhaojun Lu, Zequn Wang, Jiarui Chen, Tianyou Chen and Hao Li analyze the data. Jie Jiang wrote and revised the manuscript. Chong Liu and Xinli Zhan revised the manuscript. All authors read and approved the final manuscript.

\section{Acknowledgements}

Thanks to all co-authors who contributed to this research.

\section{References}

1. Grodahl LH, Fawcett L, Nazareth M, Smith R, Spencer S, Heneghan N, Rushton A. Diagnostic utility of patient history and physical examination data to detect spondylolysis and spondylolisthesis in athletes with low back pain: A systematic review. Man Ther. 2016;24:7-17.

2. Mlyavykh S, Ludwig SC, Kepler CK, Anderson DG. Five-year results of a clinical pilot study utilizing a pedicle-lengthening osteotomy for the treatment of lumbar spinal stenosis. J Neurosurg Spine. 2018;29(3):241-9.

3. Ravindra VM, Senglaub SS, Rattani A, Dewan MC, Hartl R, Bisson E, Park KB, Shrime MG. Degenerative Lumbar Spine Disease: Estimating Global Incidence and Worldwide Volume. Global Spine J. 2018;8(8):784-94. 
4. Khor S, Lavallee DC, Cizik AM, Bellabarba C, Dagal A, Hart RA, Howe CR, Martz RD, Shonnard N, Flum DR: Hospital and Surgeon Variation in Patient-reported Functional Outcomes after Lumbar Spine Fusion: A Statewide Evaluation. Spine (Phila Pa 1976) 2019.

5. Chan AK, Sharma V, Robinson LC, Mummaneni PV. Summary of Guidelines for the Treatment of Lumbar Spondylolisthesis. Neurosurg Clin N Am. 2019;30(3):353-64.

6. Jeong H-Y, You J-W, Sohn H-M, Park S-H. Radiologic evaluation of degeneration in isthmic and degenerative spondylolisthesis. Asian spine journal. 2013;7(1):25-33.

7. Kanno H, Ozawa H, Koizumi Y, Morozumi N, Aizawa T, Ishii Y, Itoi E. Changes in lumbar spondylolisthesis on axial-loaded MRI: do they reproduce the positional changes in the degree of olisthesis observed on X-ray images in the standing position? The Spine Journal. 2015;15(6):125562.

8. Jae Yoon Chung SKK, Jung ST. Spontaneous Reduction Finding: Magnetic Resonance Imaging Evaluation of Segmental Instability in Spondylolisthesis. Asian Spine Journal. 2012;6:221 226.

9. Yizhar Floman M. Progression of Lumbosacral Isthmic Spondylolisthesis in Adults. Spine (Phila Pa 1976). 2000;25:342-7.

10. Timon SJ, Gardner MJ, Wanich T, Poynton A, Pigeon R, Widmann RF, Rawlins BA, Burke SW. Not all spondylolisthesis grading instruments are reliable. Clin Orthop Relat Res 2005(434):157-162.

11. Mobbs RJ, Phan K, Malham G, Seex K, Rao PJ. Lumbar interbody fusion: techniques, indications and comparison of interbody fusion options including PLIF, TLIF, MI-TLIF, OLIF/ATP, LLIF and ALIF. J Spine Surg. 2015;1(1):2-18.

12. Kato S, Oshima Y, Matsubayashi Y, Taniguchi Y, Tanaka S, Takeshita K. Minimum Clinically Important Difference and Patient Acceptable Symptom State of Japanese Orthopaedic Association Score in Degenerative Cervical Myelopathy Patients. Spine (Phila Pa 1976). 2019;44(10):691-7.

13. Jeong HY, You JW, Sohn HM, Park SH. Radiologic evaluation of degeneration in isthmic and degenerative spondylolisthesis. Asian Spine J. 2013;7(1):25-33.

14. Pascal Niggemann M, * Johannes Kuchta, MD,† Hans-Konrad Beyer, MD,* Grosskurth D, MD,* Thorsten Schulze, MD, $\neq$ and Karl-Stefan Delank, MD§: Spondylolysis and Spondylolisthesis Prevalence of Different Forms of Instability and Clinical Implications. Spine (Phila Pa 1976) 2010, XX:000-000.

15. Okuda S, Oda T, Yamasaki R, Haku T, Maeno T, Iwasaki M. Posterior lumbar interbody fusion with total facetectomy for low-dysplastic isthmic spondylolisthesis: effects of slip reduction on surgical outcomes: clinical article. J Neurosurg Spine. 2014;21(2):171-8.

16. Hasegawa K, Kitahara K, Shimoda H, Ishii K, Ono M, Homma T, Watanabe K. Lumbar degenerative spondylolisthesis is not always unstable: clinicobiomechanical evidence. Spine (Phila Pa 1976). 2014;39(26):2127-35.

17. Bydon M, Alvi MA, Goyal A. Degenerative Lumbar Spondylolisthesis: Definition, Natural History, Conservative Management, and Surgical Treatment. Neurosurg Clin North Am. 2019;30(3):299-304. 
18. Blumenthal C, Curran J, Benzel EC, Potter R, Magge SN, Harrington JF, Coumans J-V, Ghogawala Z. Radiographic predictors of delayed instability following decompression without fusion for degenerative Grade I lumbar spondylolisthesis. Journal of Neurosurgery: Spine. 2013;18(4):340-6.

19. Karsy M, Bisson EF. Surgical Versus Nonsurgical Treatment of Lumbar Spondylolisthesis. Neurosurg Clin N Am. 2019;30(3):333-40.

20. Smorgick Y, Mirovsky Y, Floman Y, Rand N, Millgram M, Anekstein Y. Long-term results for total lumbar facet joint replacement in the management of lumbar degenerative spondylolisthesis. Journal of neurosurgery Spine 2019:1-6.

21. Lian XF, Hou TS, Xu JG, Zeng BF, Zhao J, Liu XK, Yang EZ, Zhao C. Single segment of posterior lumbar interbody fusion for adult isthmic spondylolisthesis: reduction or fusion in situ. Eur Spine J. 2014;23(1):172-9.

22. Huang KY, Lin RM, Lee YL, Li JD. Factors affecting disability and physical function in degenerative lumbar spondylolisthesis of L4-5: evaluation with axially loaded MRI. Eur Spine J. 2009;18(12):1851-7.

23. Shillingford JN, Laratta JL, Reddy H, Ha A, Lehman RA Jr, Lenke LG, Fischer CR. Postoperative Surgical Site Infection After Spine Surgery: An Update From the Scoliosis Research Society (SRS) Morbidity and Mortality Database. Spine Deform. 2018;6(6):634-43.

\section{Tables}

Table 1-1. Baseline information.

\begin{tabular}{|c|c|c|c|c|c|c|}
\hline Group & \multirow{2}{*}{$\begin{array}{l}\text { Group } \\
\text { A1 }\end{array}$} & \multirow[t]{2}{*}{ Group B } & \multirow{2}{*}{$\begin{array}{l}P \\
\text { value }\end{array}$} & \multirow{2}{*}{$\begin{array}{l}\text { Group } \\
\text { A2 }\end{array}$} & \multirow[t]{2}{*}{ Group B } & \multirow{2}{*}{$\begin{array}{l}\mathrm{P} \\
\text { value }\end{array}$} \\
\hline Index & & & & & & \\
\hline Gender冈Male/Female) & $5 / 22$ & $13 / 39$ & 0.515 & $7 / 18$ & $13 / 39$ & 0.779 \\
\hline $\begin{array}{l}\text { Screws (pedicle screws / pulling } \\
\text { pedicle screws) }\end{array}$ & $12 / 15$ & $28 / 24$ & 0.428 & $10 / 15$ & $28 / 24$ & 0.255 \\
\hline Slip section (L3 / L4 / L5) & $2 / 14 / 11$ & $0 / 30 / 22$ & 0.137 & $0 / 16 / 9$ & $0 / 30 / 22$ & 0.597 \\
\hline Slip indexing $\left(\nabla^{\circ} / \nabla^{\circ} / \nabla^{\circ}\right)$ & $15 / 10 / 2$ & $34 / 18 / 0$ & 0.125 & $16 / 9 / 0$ & $34 / 18 / 0$ & 0.905 \\
\hline Isthmus fissure / degeneration & $17 / 10$ & $36 / 16$ & 0.574 & $19 / 6$ & $36 / 16$ & 0.538 \\
\hline Spinal stenosis (with / without) & $20 / 7$ & $32 / 20$ & 0.203 & $12 / 13$ & $32 / 20$ & 0.154 \\
\hline Erosive discitis (yes / no) & $11 / 16$ & $26 / 26$ & 0.759 & $10 / 15$ & $26 / 26$ & 0.615 \\
\hline Number of fusion segments $(1 / 2)$ & $21 / 6$ & $34 / 18$ & 0.256 & $21 / 4$ & $34 / 18$ & 0.184 \\
\hline
\end{tabular}

Table 1-1. Baseline information. Group A1 represents the unstable slip group, group A2 represents the unstable angle group, and group B represents the stable group. 
Table 1-2 Baseline information.

\begin{tabular}{|lllllll|}
\hline Group & Group A1 & Group B & & Group A2 & Group B & \\
Index & $\begin{array}{l}\text { Mean } \pm \\
\text { standard } \\
\text { deviation }\end{array}$ & $\begin{array}{l}\text { Mean } \pm \\
\text { standard } \\
\text { deviation }\end{array}$ & $\begin{array}{l}\text { value } \\
\text { van }\end{array}$ & $\begin{array}{l}\text { Mean } \pm \\
\text { standard } \\
\text { deviation }\end{array}$ & $\begin{array}{l}\text { Mean } \pm \\
\text { standard } \\
\text { deviation }\end{array}$ & value \\
$\begin{array}{l}\text { Course of } \\
\text { disease (y) }\end{array}$ & $6.38 \pm 6.37$ & $5.50 \pm 6.90$ & 0.552 & $5.81 \pm 7.52$ & $5.50 \pm 6.90$ & 0.857 \\
BMl『kg/m2】 & $22.51 \pm 3.1$ & $23.65 \pm 3.41$ & 0.147 & $22.47 \pm 3.0$ & $23.65 \pm 3.41$ & 0.146 \\
$\begin{array}{l}\text { Day of } \\
\text { hospitalization } \\
\text { (d) }\end{array}$ & $12.56 \pm 4.80$ & $11.98 \pm 5.93$ & 0.834 & $10.52 \pm 4.60$ & $11.98 \pm 5.93$ & 0.282 \\
$\begin{array}{l}\text { Operation time } \\
\text { (min) }\end{array}$ & $185.8 \pm 76.9$ & $166.9 \pm 57.6$ & 0.224 & $157.2 \pm 56.6$ & $166.9 \pm 57.6$ & 0.51 \\
$\begin{array}{l}\text { Bleeding } \\
\text { volume (ml) }\end{array}$ & $653.7 \pm 452.5$ & $637.5 \pm 453.6$ & 0.881 & $526.0 \pm 400.8$ & $637.5 \pm 453.6$ & 0.298 \\
$\begin{array}{l}\text { Follow-up time } \\
\text { (m) }\end{array}$ & $33.41 \pm 7.10$ & $31.17 \pm 7.00$ & 0.184 & $2.88 \pm 7.51$ & $31.17 \pm 7.00$ & 0.331 \\
\hline
\end{tabular}

Table 1-2 Baseline information. Remarks: Group A1: unstable slip group, Group A2: unstable angle group, Group B: stable group, inspection level $a=0.05, y$ : year, d: day, min: minute, ml: milliliter, m: month.

\section{Figures}




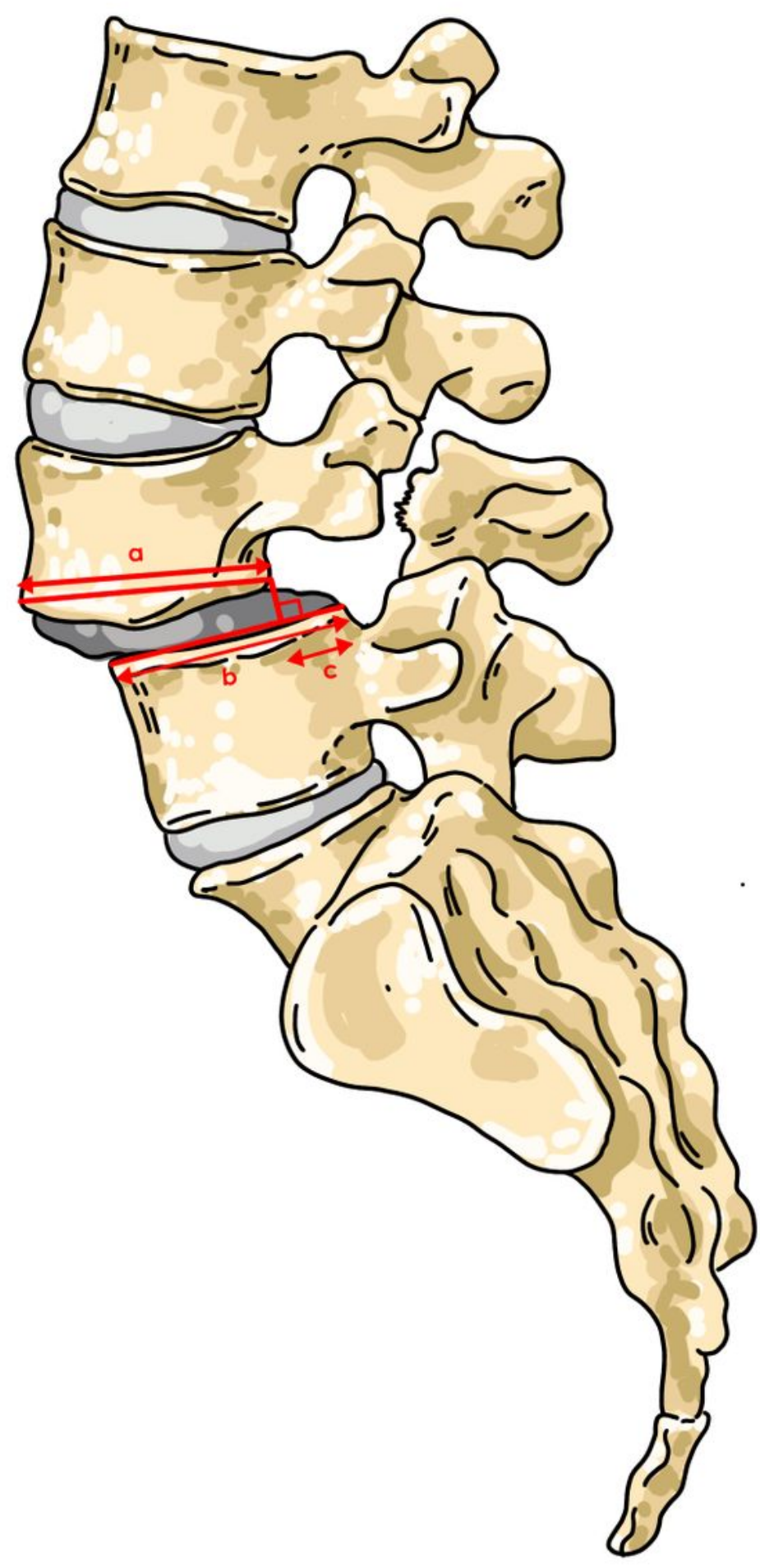

\section{Figure 1}

Schematic diagram of radiographic index measurements. Letter a represents the width of the lower endplate line of the spondylolisthesis vertebra, letter b represents the width of the upper endplate line of the lower vertebral body of the spondylolisthesis, and letter $\mathrm{c}$ represents the intersection of the posterior angle of the spondylolisthesis and the vertical line of the upper vertebral body The distance from the 
posterior superior angle of the lower vertebral body. Translation Distances (TD) $=\mathrm{c}$; slip rate $(\mathrm{SR})=\mathrm{c} / \mathrm{b} \times$ $100 \%$; segment angle $(S A)=$ angle between $a$ and $b$.

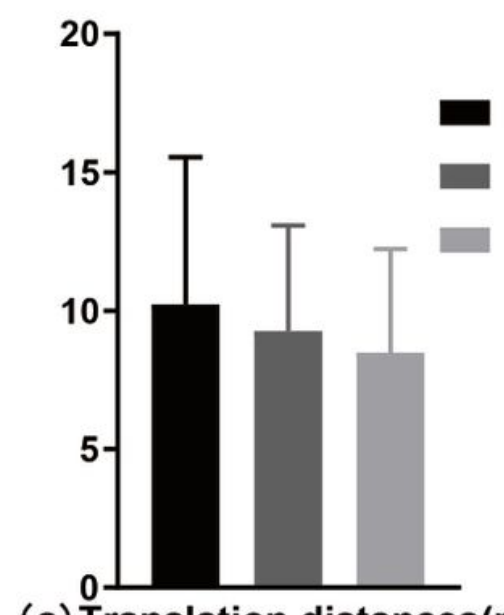

(a) Translation distances $(\mathrm{mm})$

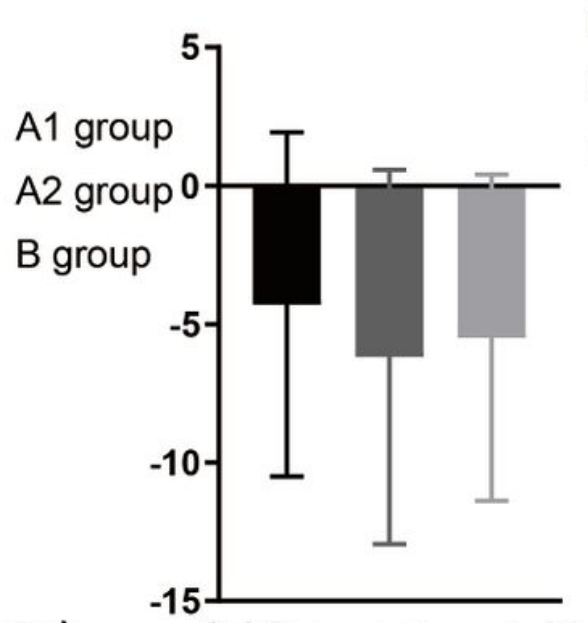

(b) Segment angle $\left({ }^{\circ}\right)$
A1 group

A2 group $\left.{ }^{50}\right]$ A1 group

B group

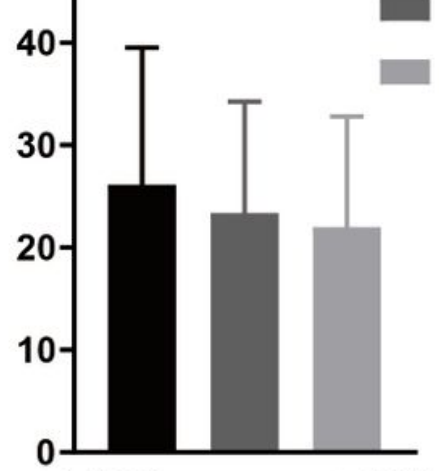

(c) Slippage rate(\%) A2 group B group

Figure 2

Preoperative imaging parameters. The graph (a) shows the preoperative slip distance of each group. The graph (b) shows the preoperative segment angle of each group. The graph (c) shows the preoperative slip rate for each group.
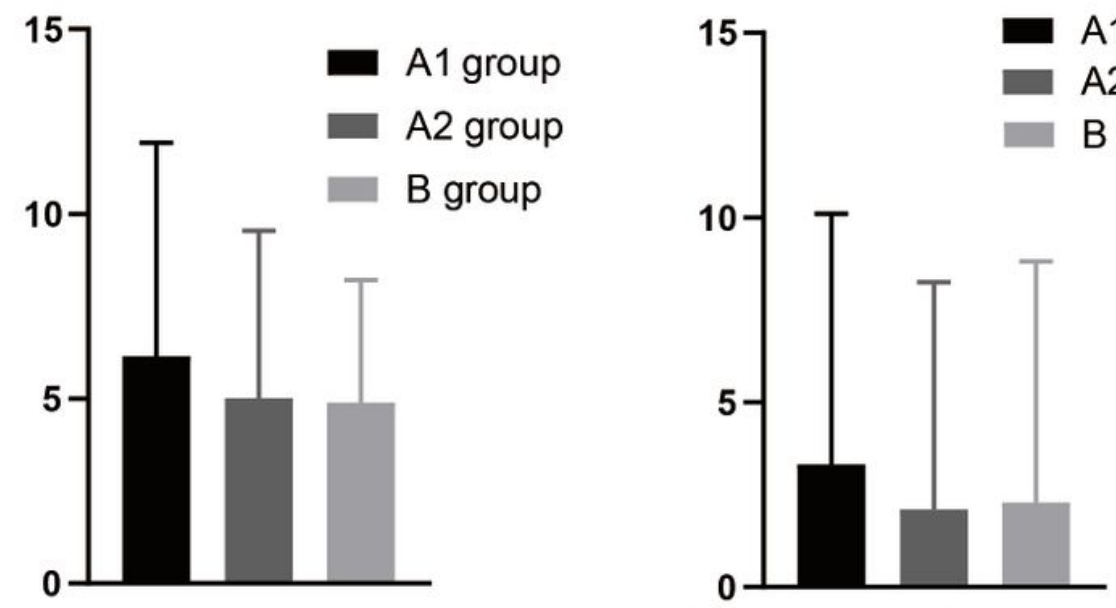

(a) Translation distances correction $(\mathrm{mm})(\mathrm{b})$ Segment angle correction $\left({ }^{\circ}\right)$

A1 group

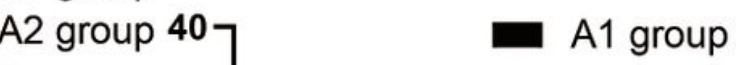

B group

A2 group

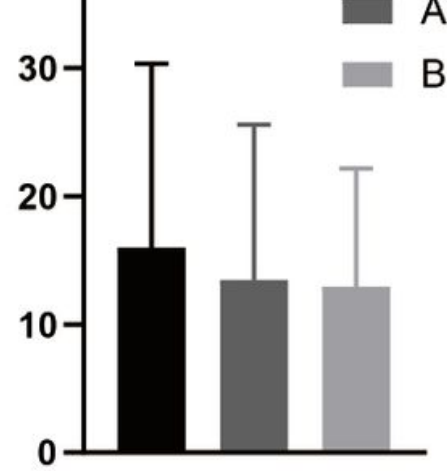

(c)Slippage correction(\%)

Figure 3

Postoperative imaging parameters. The graph (a) shows the postoperative slip distance correction of each group. The graph (b) the postoperative segment angle correction of each group. The graph (c) the postoperative slip rate correction for each group. 

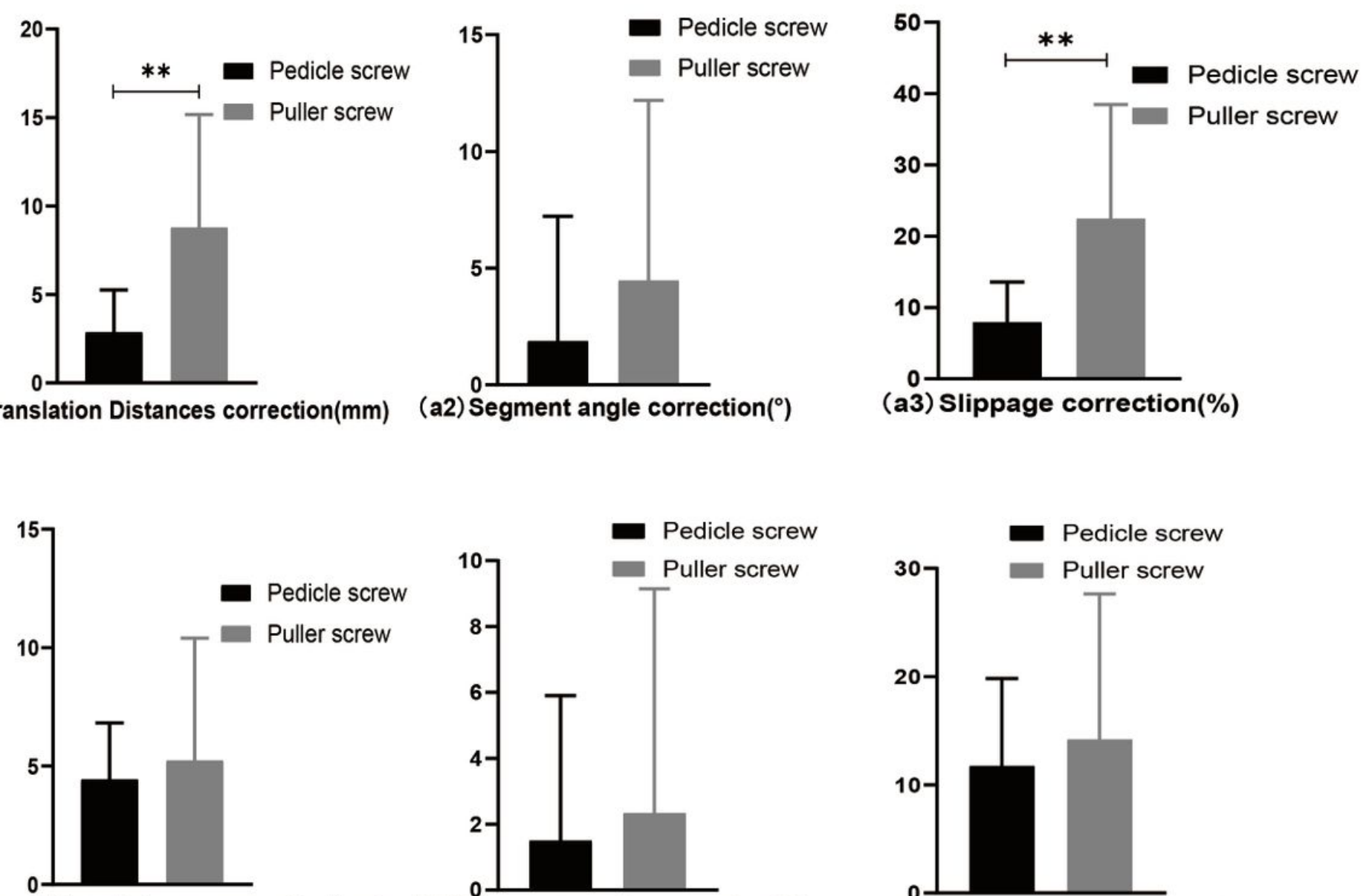

(b1) Translation distances correction(mm) (b2) Segment angle correction(\%)
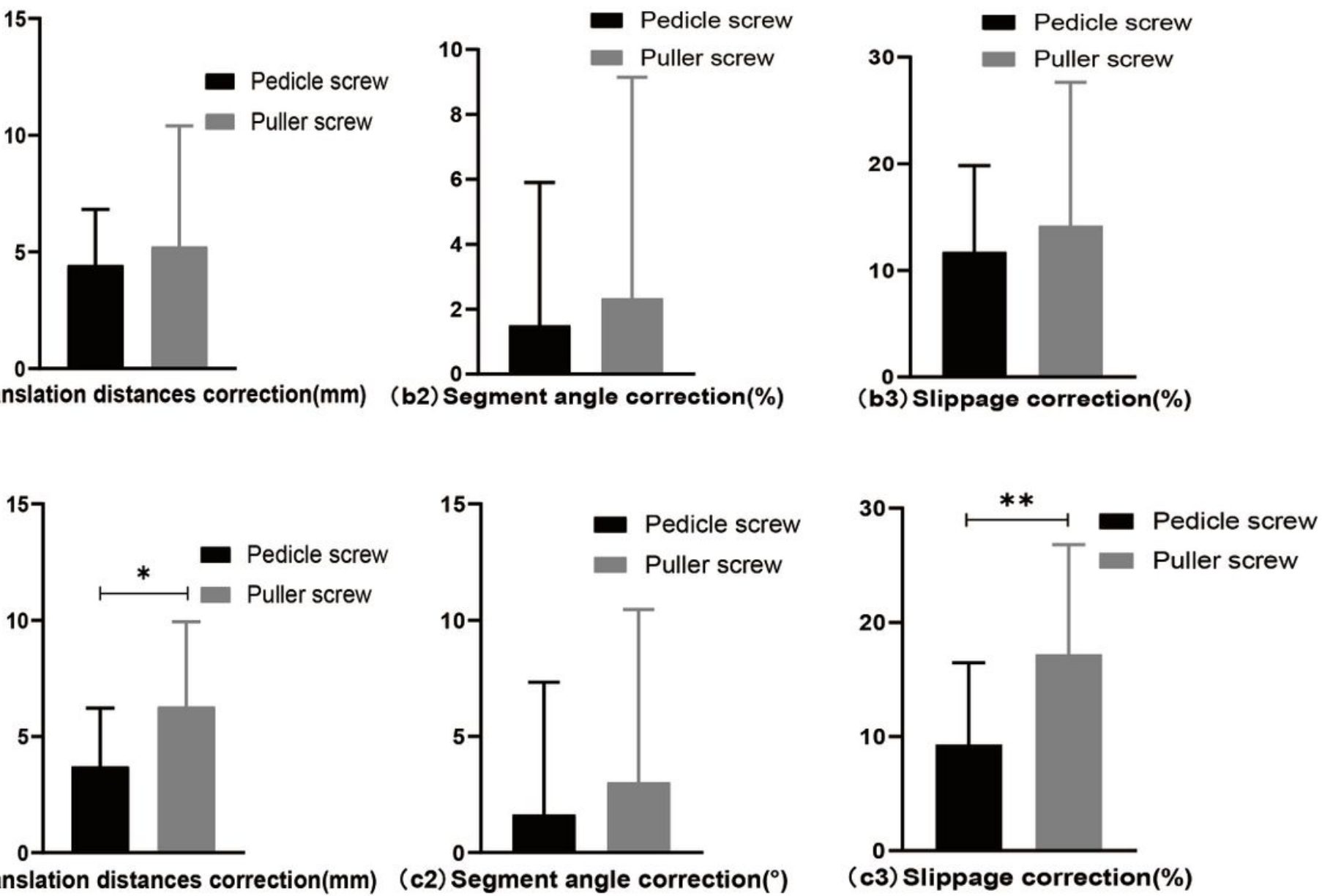

(c1) Translation distances correction( $\mathrm{mm})$ (c2) Segment angle correction $\left({ }^{\circ}\right)$

Figure 4

The use of the pedicle screw and the puller screw. * represents a P-value $<0.05$, ** represents a P-value $<0.01$. (a1) The diagram shows the translation distances correction for group A1 using pedicle screws and using puller screws. (a2) The diagram shows the segment angle correction for group A1 using pedicle screws and using puller screws. (c3) The diagram shows the slippage correction for group A1 using pedicle screws and using puller screws. (b1) The diagram shows the translation distances correction for group A2 using pedicle screws and using puller screws. (b2) The diagram shows the 
segment angle correction for group A2 using pedicle screws and using puller screws. (b3) The diagram shows the slippage correction for group A1 using pedicle screws and using puller screws. (c1) The diagram shows the translation distances correction for group B using pedicle screws and using puller screws. (c2) The diagram shows the segment angle correction for group B using pedicle screws and using puller screws. (c3) The diagram shows the slippage correction for group A1 using pedicle screws and using puller screws.

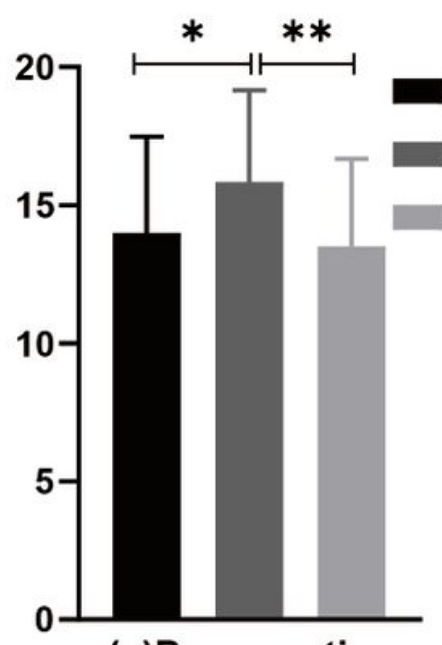

(a)Preoperative

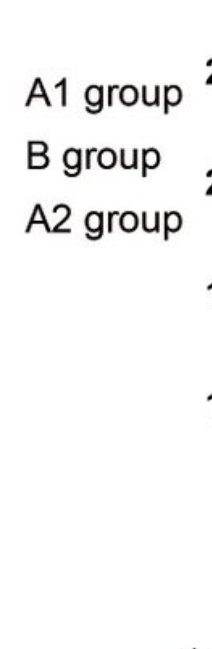

(b) One month after surgery

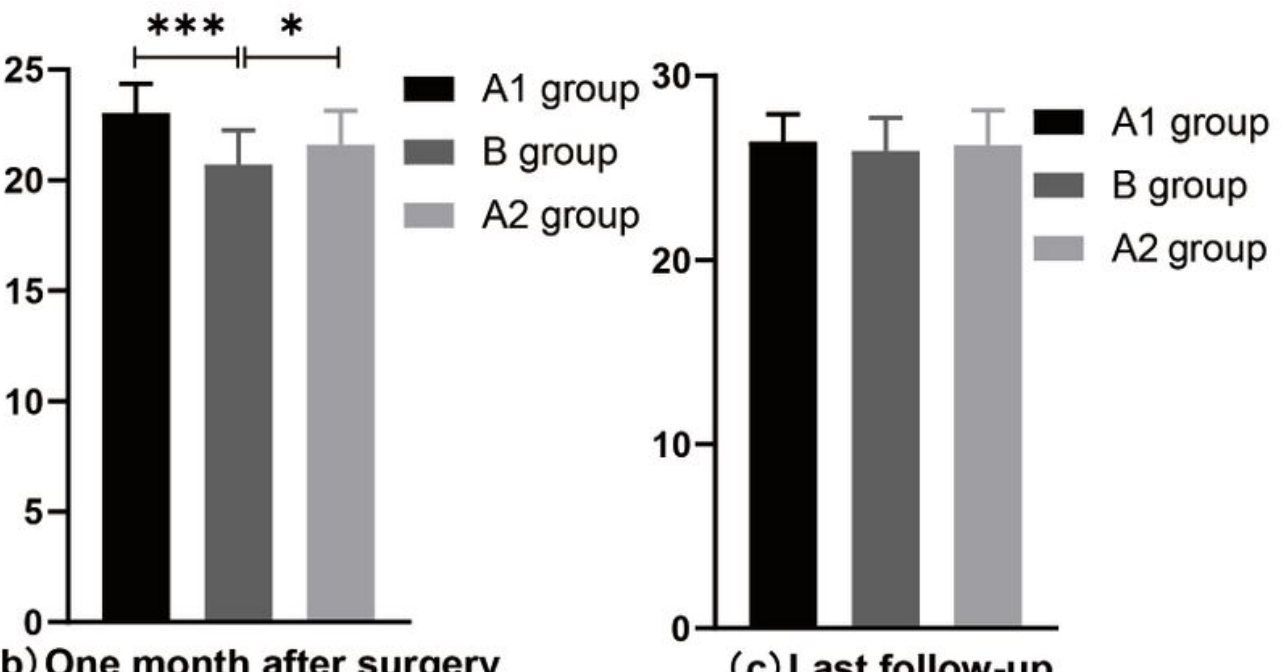

(c) Last follow-up

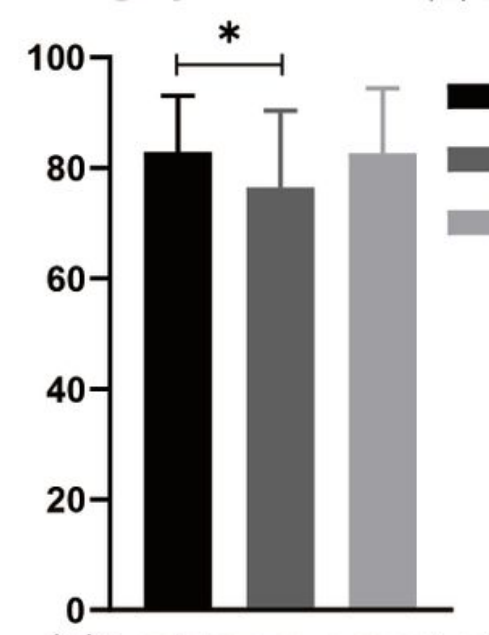

(e) Last improvement rate

\section{(d) One month improvement rate}

\section{Figure 5}

Preoperative JOA score status of each group. * represents a $p$ value $<0.05$, ** represents a $p$ value $<0.01$. The (a) to (e) plots show the JOA score status of preoperative, one-month postoperative, final follow-up, one-month improvement rate, and final improvement rate, respectively. 

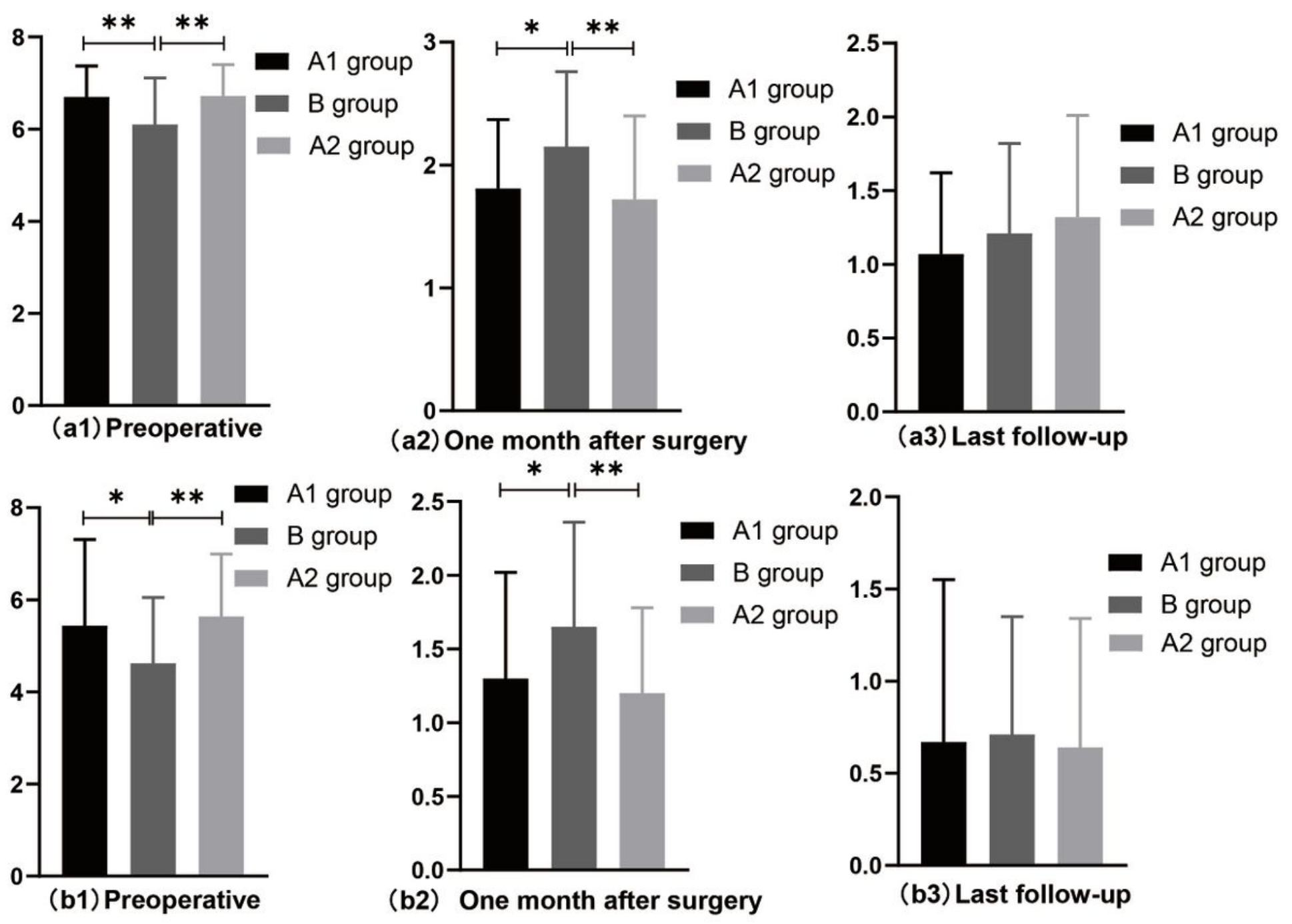

Figure 6

The VAS scores for each group. * represents a p value $<0.05, * \star$ represents a p value $<0.01$. The graph (a1) to graph (a3) represent the VAS score status of low back pain before surgery, one month after surgery, and at the last follow-up visit, respectively. The graph (b1) to graph (b3) represent the VAS score status of leg pain before surgery, one month after surgery, and at the last follow-up visit, respectively. 


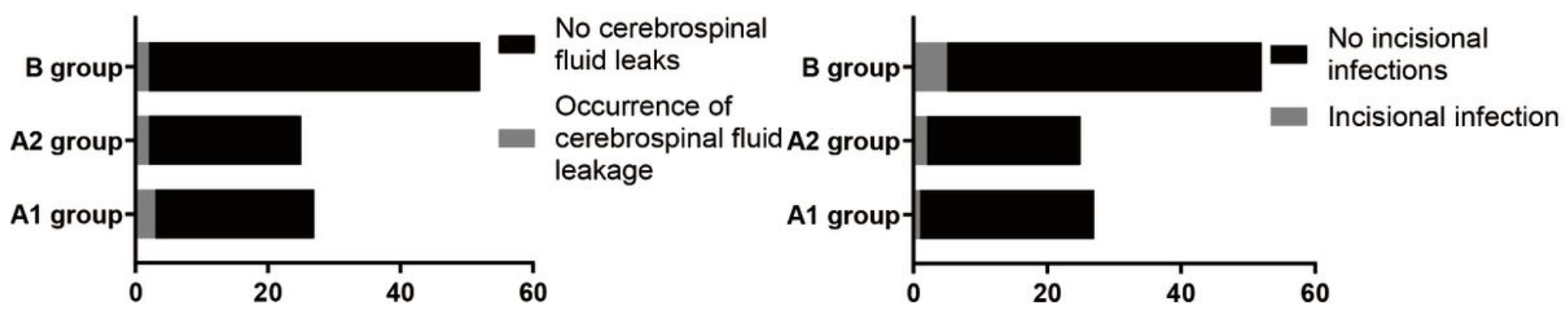

(a) cerebrospinal fluid leakage

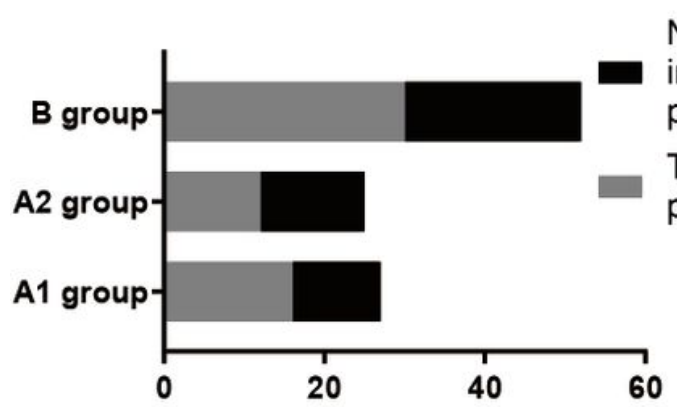

No temporary

intestinal paralysis

Temporary intestinal paralysis occurs

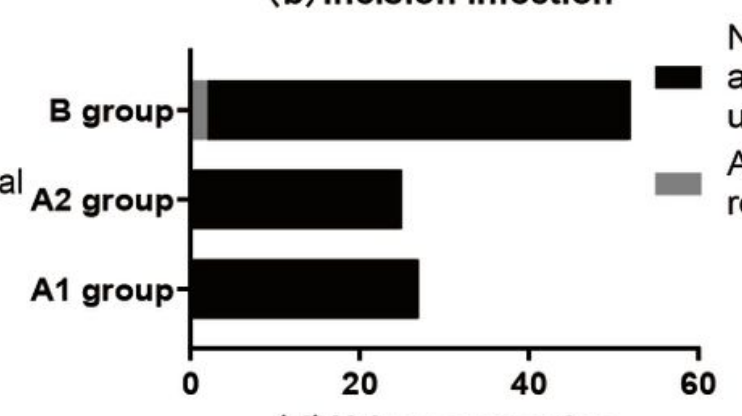

Not

accompanied by urine retention Associated urine retention

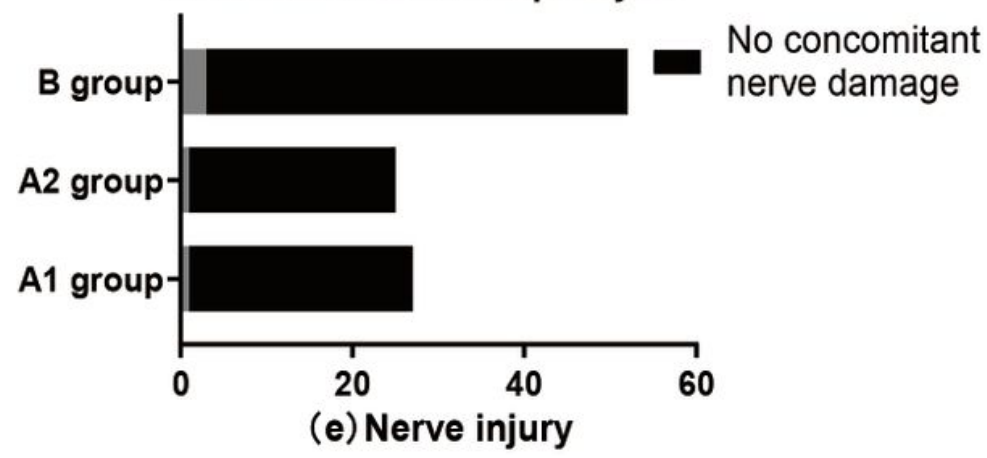

Figure 7

Complications occurred in each group. Figures (a) to (e) show the occurrence of cerebrospinal fluid leakage, incisional infection, temporary intestinal paralysis, urinary storage and nerve damage, respectively. 